\title{
(2-Fluoroallyl)boration of ketones with (2-fluoroallyl)boronates
}

Maxim A. Novikov ${ }^{1 *}$, Angelina Yu. Bobrova ${ }^{1,2}$, Igor A. Mezentsev ${ }^{1,2}$, Michael G. Medvedev ${ }^{1}$ and Yury V. Tomilov ${ }^{1}$

${ }^{1}$ N.D. Zelinsky Institute of Organic Chemistry, Russian Academy of Sciences, 47 Leninsky prosp., 119991 Moscow, Russian Federation

${ }^{2}$ Higher Chemical College of the Russian Academy of Sciences, D. I. Mendeleev University of Chemical Technology of Russia, 9 Miusskaya pl., 125047 Moscow, Russian Federation Fax: +7499 13563 90; e-mail: manovikov@ioc.ac.ru

Fig. S1. Single crystal X-ray structure of compound 5. Atoms are represented as thermal ellipsoids ( $p=50 \%)$

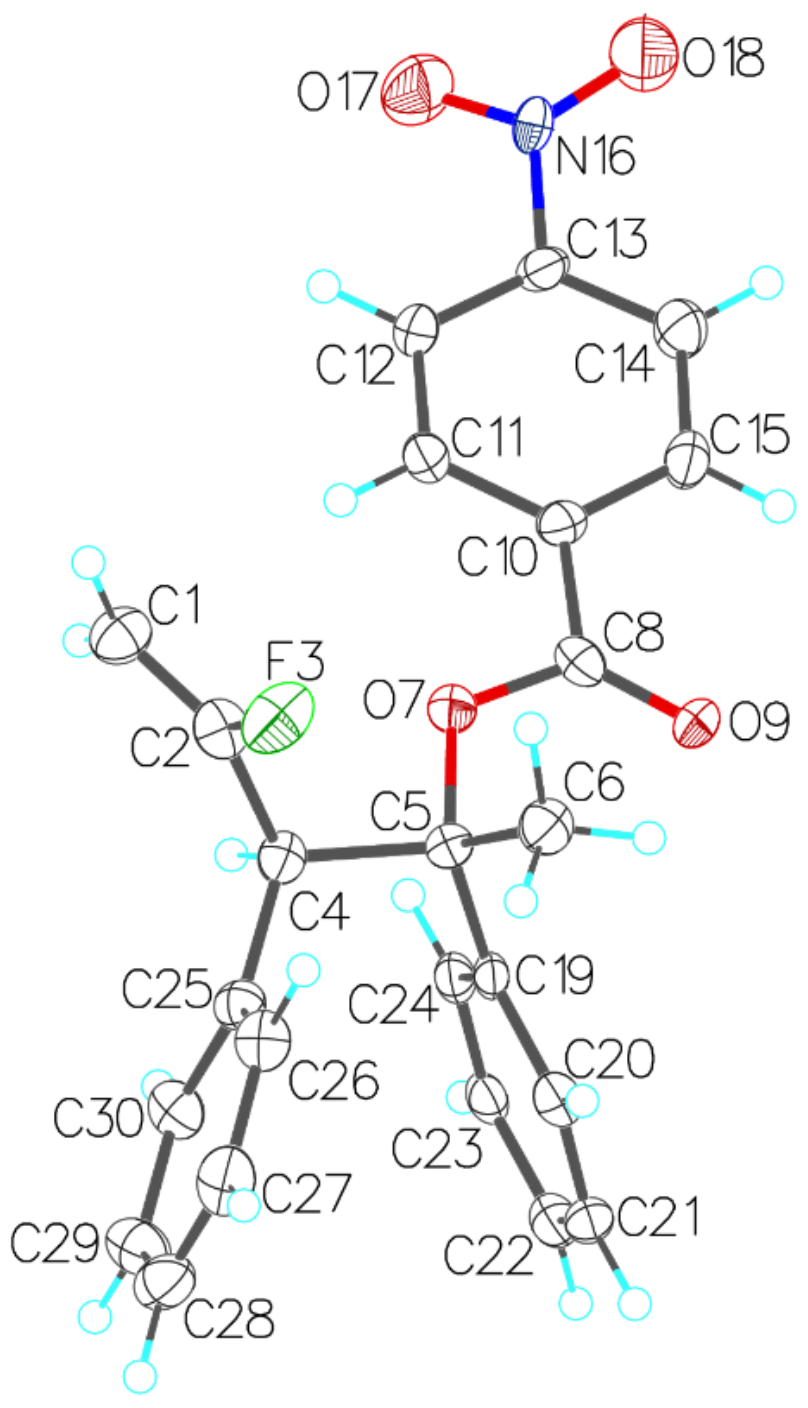


X-ray diffraction data for compound $\mathbf{5}$ was collected on a SMART APEX II area-detector diffractometer (graphite monochromator, $\omega$-scan technique), using MoK $\alpha$-radiation (0.71073 $\AA$ ). The intensity data were integrated by the SAINT program ${ }^{1}$ and were corrected for absorption and decay using SADABS ${ }^{2}$. All structures were solved by direct methods using SHELXS ${ }^{3}$, and were refined on $\mathrm{F}^{2}$ using SHELXL-2014/2017 ${ }^{4}$. All non-hydrogen atoms were refined with anisotropic displacement parameters. All hydrogen atoms were placed in ideal calculated positions and refined as riding atoms with relative isotropic displacement parameters taken as $\mathrm{U}_{\text {iso }}(\mathrm{H})=1.5 \mathrm{U}_{\mathrm{eq}}(\mathrm{C})$ for methyl groups and $\mathrm{U}_{\mathrm{iso}}(\mathrm{H})=1.2 \mathrm{U}_{\mathrm{eq}}(\mathrm{C})$ for the rest ones. Crystal data and structure refinement parameters can be retrieved free of charge from www.ccdc.cam.ac.uk/conts/retrieving.html (CCDC 1988716).

Table S1. Crystal data and structural refinement details for compound $\mathbf{5}$

\begin{tabular}{|c|c|}
\hline \multicolumn{2}{|l|}{ Crystal data } \\
\hline Chemical formula & $\mathrm{C}_{24} \mathrm{H}_{20} \mathrm{FNO}_{4}$ \\
\hline$M_{\mathrm{r}}$ & 405.41 \\
\hline Crystal system, space group & Orthorhombic, $P 2_{1} 2_{1} 2_{1}$ \\
\hline Temperature $(\mathrm{K})$ & 120 \\
\hline$a, b, c(\AA)$ & $6.0732(13), 11.111(2), 29.291(6)$ \\
\hline$V\left(\AA^{3}\right)$ & $1976.5(7)$ \\
\hline$Z$ & 4 \\
\hline Radiation type & Mo K $\alpha$ \\
\hline$\mu\left(\mathrm{mm}^{-1}\right)$ & 0.10 \\
\hline Crystal size $(\mathrm{mm})$ & $0.3 \times 0.2 \times 0.2$ \\
\hline \multicolumn{2}{|l|}{ Data collection } \\
\hline Diffractometer & Bruker APEX-II CCD \\
\hline Absorption correction & $\begin{array}{l}\text { Multi-scan. SADABS2014/5 (Bruker,2014/5) was used for } \\
\text { absorption correction. wR2(int) was } 0.0819 \text { before and } \\
0.0728 \text { after correction. The Ratio of minimum to } \\
\text { maximum transmission is } 0.8217 \text {. The } \lambda / 2 \text { correction } \\
\text { factor is } 0.00150 \text {. }\end{array}$ \\
\hline$T_{\min }, T_{\max }$ & $0.612,0.745$ \\
\hline $\begin{array}{l}\text { No. of measured, independent and } \\
\text { observed }[I>2 \sigma(I)] \text { reflections }\end{array}$ & $10861,3486,2165$ \\
\hline$R_{\text {int }}$ & 0.118 \\
\hline$(\sin \theta / \lambda)_{\max }\left(\AA^{-1}\right)$ & 0.595 \\
\hline \multicolumn{2}{|l|}{ Refinement } \\
\hline$R\left[F^{2}>2 \sigma\left(F^{2}\right)\right], \mathrm{w} R\left(F^{2}\right), S$ & $0.055,0.123,0.98$ \\
\hline No. of reflections & 3486 \\
\hline No. of parameters & 273 \\
\hline H-atom treatment & H-atom parameters constrained \\
\hline$\Delta \rho_{\max }, \Delta \rho_{\min }\left(\mathrm{e} \AA^{-3}\right)$ & $0.21,-0.25$ \\
\hline Absolute structure & Refined as an inversion twin. \\
\hline Absolute structure parameter & $-1(2)$ \\
\hline
\end{tabular}


(1) Bruker. APEXII; Bruker AXS Inc.: Madison, Wisconsin, USA, 2008, 2008.

(2) Sheldrick, G. M. SADABS. Program for Empirical Absorption Correction; University of Gottingen, Germany, 1997.

(3) Sheldrick, G. M. Acta Crystallographica Section A Foundations of Crystallography 2008, 64, 112-122.

(4) Sheldrick, G. M. Acta Crystallographica Section C Structural Chemistry 2015, 71, 3-8. 